\title{
Analysis of bilateral breast carcinomas: a profile of patients at a reference service
}

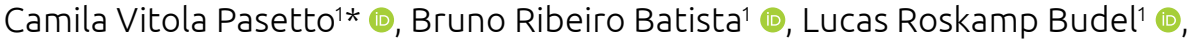 \\ Mariana De Nadai Andreoli ${ }^{10}$, Vinicius Milani Budel ${ }^{1}$ (1)
}

\section{ABSTRACT}

Objective: To select cases of bilateral breast carcinoma (BBC) of patients seen at Hospital de Clínicas of Paraná, besides recognizing clinical and family characteristics, histological and immunohistochemical pattern, and incidences of synchronic/metachronic tumor in these patients. Method: Observational and analytical study of BBC cases of patients treated at Hospital de Clínicas of Paraná, from 2003 to 2019, developed from the analysis of medical records. Result: A total of 42 patients with BBC were selected. The incidence of BBC was $3.64 \%$. All patients were women, mostly of white skin color and postmenopausal, with an average age of 51.82 years. Half patients showed a positive family history for cancer, with breast cancer present in $46 \%$, ovarian cancer in $16 \%$, and other topographies in $68 \%$. In this sample, the synchronous tumor was present in $55 \%$ of patients, and the metachronous tumor, in $45 \%$. Regarding patients' initial clinical staging, $61 \%$ had a locally advanced tumor at diagnosis. Both in the group of synchronic and metachronic tumors, the ductal subtype was the most frequent. Regarding the immunohistochemical subtype, patients in both groups had Luminal B tumors more frequently. In the group of metachronic tumors, the average time between the diagnosis of the first tumor and the second tumor was 5.68 years. Conclusion: In this sample, BBC is associated with a relevant family history, with a synchronic presentation pattern, from histology to ductal and immunohistochemistry to Luminal B as the most frequent.

KEYWORDS: Breast neoplasms; Synchronous neoplasm; Metachronous neoplasm.

\section{INTRODUCTION}

Bilateral breast cancer $(\mathrm{BBC})$ is a rare clinical entity. Its estimated incidence is between $0.3 \%$ and $12 \% .^{1}$ This neoplasm pattern can be considered synchronous, when it occurs simultaneously, or metachronous, when it is diagnosed from one month to a year after the primary tumor is found. ${ }^{2,3}$

The importance of studying BBC is due to the increased incidence of cases of breast carcinoma and its early diagnosis which increases the survival time for these patients. However, the risk of developing contralateral breast cancer (CBC) is also increased. Patients who had early breast cancer treated have from two to six times greater chance of developing the contralateral neoplasia than the female population in general. The estimated risk is $0.4 \%$ to $0.8 \%$ per survival year. ${ }^{4}$

The relevance of BBC was first studied in 1956. The study showed that patients who treated breast cancer had from three to four times greater chance of developing bilateral cancer, which behaves as a primary tumor and not metastatic. ${ }^{5}$

There are several risk factors for bilateral breast cancer. Among them, the histological and immunohistochemical type, family history of breast cancer, genetic mutations, and age at diagnosis of the first cancer are the most important. ${ }^{6,7}$

The histological type most frequently associated with bilateral breast cancer is the lobular one. In the literature, the risk ranges from 1.42 to 6.55. According to the authors, this variation is due to the difference in biological behavior and tumor etiology. ${ }^{8,9}$

Family history is relevant in the following situations: a first or second degree family member with breast cancer before the age of 45, or two or more of these family members with this type of cancer before the age of 50; a family member with two or more breast cancers; an individual with ovarian, fallopian tube, or primary peritoneal cancer; male breast cancer; or three or more family members with cancer in the following types and/or topographies (especially if diagnosed at the age of 50 or before that): breast, pancreas, prostate (metastatic Gleason score 7), melanoma, sarcoma, adrenocortical carcinoma, brain tumors, leukemia, colon, endometrium, thyroid, kidney, hamartomatous polyps of the gastrointestinal tract cancer, and an individual of Ashkenazi Jewish origin with breast, ovarian, or pancreas cancer at any age. ${ }^{10}$ 
As to family history, the relative risk (RR) of increase in $\mathrm{BBC}$ was 2.8 , especially for first-degree family members. ${ }^{11} \mathrm{~A}$ study by Reiner et al. from 2013 showed that the risk of contralateral breast cancer for a 30- to 34-year-old patient with breast cancer without BRCA1 and 2 mutations and no family history is $7 \%$ in 10 years. Patients without genetic mutations, but with a second-degree relative with breast cancer, are at 9\% risk; those with an affected first-degree relative have a $14.7 \%$ risk of contralateral breast cancer. A bilaterally affected family member increases the risk of a patient without a genetic mutation for contralateral breast cancer to $23.7 \%{ }^{12}$

Bilaterality suggests genetic origin, that is, hereditary breast cancer. There are pathogenic mutations associated with this type of cancer, especially in BBC, which are: BRCA1 and BRCA2 (50\%-85\%), PALB2 (33\%-58\%), TP53 (Li-Fraumeni syndrome, 50\%-90\%), PTEN (Cowden syndrome/PTEN Hamartoma Tumor Syndrome, 25\%-50\%), STK11 (32-54\%), and CDH1 (30\%-50\%). The most important mutation related to bilateral neoplasm is BRCA1 and BRCA2. A population study with 705 women with $\mathrm{BBC}$ - with a mutation in the BRCA 1 and 2 genes - showed that the risk of bilateral neoplasia was 4.5 and 3.4 in BRCA 1 and 2 mutations, respectively, and the estimated cumulative risk over 10 years was 18.4 with the mutation and 4.8 without it. ${ }^{13}$

The patient's age at diagnosis of the primary tumor is a significant factor for bilateral cancer, especially for patients under 50 years old. ${ }^{14}$ In a study carried out in Sweden with 1,351 cases, patients over 50 years old had an RR of 1 , whereas those under 50 had an RR of $9.9 .^{15}$

The objective of the present study was to assess the clinical, familial, histological, and immunohistochemical pattern of patients with bilateral breast cancer for a better understanding of this clinical entity, which, although rare, is of great importance.

\section{METHODOLOGY}

This is a cross-sectional, retrospective, observational, and analytical study. The target population analyzed is patients treated by the tocogynecology service of Hospital de Clínicas of Universidade Federal do Paraná, from January 2003 to December 2019. Patients with unilateral breast carcinoma, breast cancer whose histology did not confirm breast carcinoma, breast cancer resulting from metastasis from another primary site, and patients with information reported in their medical records in an incomplete, inconsistent, incomprehensible, or misplaced medical record were excluded.

Based on the analysis of medical records, data relating to clinical and family characteristics, histological and immunohistochemical pattern, time of diagnosis of contralateral neoplasia (synchronic/metachronic), and the type of treatment used in metachronic tumors were obtained and recorded. After that, data were grouped into spreadsheets in Microsoft Office Excel ${ }^{\circledR}$ (2016), with subsequent data analysis by the researchers.
Research waives the Free and Informed Consent Term because it is a project with simple analysis of medical record data, without direct or minimal interference in patients.

The present study was approved by the Research Ethics Committee of Hospital de Clínicas, Universidade Federal do Paraná, with Presentation Certificate for Ethical Appreciation (CAAE) No. 11701819.9.0000.0096.

\section{RESULTS}

A total of 42 patients with $\mathrm{BBC}$ was selected out of 1,523 patients seen at the tocogynecology service of Hospital de Clínicas of Universidade Federal do Paraná, of which four were excluded due to lost medical records or incomplete information on them. The incidence of BBC in the surveyed period was $3.64 \%$. All patients are women with a mean age of 51.82 years. White skin color is the most prevalent $(82 \%)$, followed by parda (11\%), and black ( $8 \%)$. The mean menarche age of patients was 12.89 , ranging from 10 to 18 years old. As to menopausal status, $42 \%$ are pre-menopausal and $58 \%$ post-menopausal, with an average age of menopause of 48, ranging from 39 to 56. Regarding pregnancy, $16 \%$ of the patients are nulligravida, $8 \%$ had one pregnancy, $32 \%$ had two pregnancies, and $45 \%$, three or more. Half patients have a positive family history for neoplasm, with breast cancer present in $46 \%$, ovarian cancer in $16 \%$, and neoplasms of other topographies in 68\%. Neoplasms of other topographies are distributed as follows: gastrointestinal tract with $21 \%$, non-ovarian gynecological with $16 \%$, urological with $16 \%$, hematological with $11 \%$, and head and neck with $5 \%$. Smoking history was present in $29 \%$ of patients, with an average burden of tobacco-related conditions of 27.36. Patients' mean body mass index (BMI) was 28.08 .

In this sample, the synchronous tumor was present in $55 \%$ of patients, whereas the metachronous tumor, in 45\%. Regarding the patients' initial clinical staging, $61 \%$ presented with locally advanced tumor (stage IIb) at their first medical appointment.

Exclusively to the group of synchronous tumors, the mean age of patients was 52.14, distributed as follows: less than 40, 14\%; between 40 and 49, 38\%; between 50 and 59, 19\%; older than 60 , $29 \%$. The ductal histological type was the most frequent (93\%), followed by the lobular type (7\%). Of the patients, $60 \%$ had moderately differentiated tumors. With respect to immunohistochemical subtype, most patients had luminal B tumors (43\%), followed by HER2 (29\%), triple negative (24\%), and luminal A (5\%). Comparing the histological and immunohistochemical profile of each breast, $62 \%$ agreed and $48 \%$ were not the same.

Exclusively to the group of synchronous tumors, the mean age of patients was 51.41, distributed as follows: less than 40,24\%; between 40 and 49, 12\%; between 50 and 59, 47\%; older than $60,18 \%$. The average time between the diagnosis of the first tumor and the appearance of the second was 5.68 years. The most common histological type was ductal carcinoma in $73 \%$, followed by lobular carcinoma in $11 \%$, medullary carcinoma in $9 \%$, and metaplastic carcinoma in 
7\%. Regarding the immunohistochemical profile, the most prevalent was luminal B in $32 \%$, luminal A in $29 \%$, triple negative in $24 \%$, and HER2 in 15\%. The histological and immunohistochemical profile of each breast was equal in only $29 \%$ of patients, who had a triple negative in $60 \%$ and luminal B in the other $40 \%$. When assessing treatment in the primary tumor, $41 \%$ of patients underwent neoadjuvant therapy ( $86 \%$ with standard chemotherapy and $14 \%$ hormone therapy), $53 \%$ underwent conservative surgery, and $73 \%$, axillary lymphadenectomy. Of the patients, $67 \%$ had their tumors irradiated, and $87 \%$ performed adjuvant therapy according to their tumor profile.

\section{DISCUSSION}

Bilateral breast carcinomas (BBC) are rare cancer events. In the present study, despite the small sample, half patients have a positive family history from the oncological point of view, of which $46 \%$ are in breast topography and $16 \%$, in ovarian topography, reiterating the importance of this risk factor, which has been well described in the literature. ${ }^{10,11}$

In research, 55\% are synchronous tumors. Upon diagnosis, neoplasm showed to be locally advanced, that is, above stage IIb. On the other hand, synchronic cancer represents $1 \%$ of the total, and metachronic cancer is seven times more frequent in the literature. ${ }^{16}$ This is probably due to the small sample size and the quality of the health system offered to this selected group.

Regarding patients' age, the trend in the two groups is different, although the average age is quite similar. In the synchronic ones, $52 \%$ of the sample is made up of women under 50 years old, whereas in the metachronic ones, $65 \%$ was above that age.

As for the histological subtype and the tumor grade, the study results were like those found in unilateral carcinomas. Both in the synchronous and metachronic groups, positive hormone receptor tumors were the most frequent. In the literature, the profile of the highest risk for bilateral breast cancer is that of negative hormone receptors, as in a study with 4,036 patients who presented that the risk of developing another tumor bilaterally was 10 times greater in negative receptors. ${ }^{17}$

Besides that, in the synchronic group, $52 \%$ of the patients had HER2 or triple negative tumors, that is, those potentially more aggressive tumors, whereas in the metachronic group the immunohistochemical profile was similar to the distribution of unilateral breast tumors. The aggressiveness and the worse prognosis of bilateral tumors is described in other articles. Bilateral tumors have lower survival disease-free, and high rates oflymphatic spread and distant metastasis. ${ }^{18}$ According to a study carried out with 1,705 patients, the rates oflocal recurrence in five and 10 years were $4.5 \%$ and $9.1 \%$, respectively, for patients with bilateral cancer; versus $3.3 \%$ and $7.6 \%$, respectively, for unilateral cancer. In 10 years, the rates of distant metastases were $26.9 \%$ and $50.7 \%$ for unilateral and bilateral cancer, respectively. Survival in five and 10 years was $82.1 \%$ and $41 \%$ in patients with bilateral cancer, respectively, and $91.4 \%$ and $84 \%$ for unilateral cases. ${ }^{16}$
When comparing the samples from each breast in the metachronous group, most were discordant in relation to the histological and immunohistochemical profile. This generates an interesting caveat which is that when treating a bilateral tumor, we must often approach it as a second primary tumor.

Although this is a rare pathology, there is a description of an important tool to prevent the development of BBC in the literature: contralateral risk-reducing mastectomy. However, this is beneficial only for high-risk patients regarding the development of BBC, which includes patients with known BRCA1, BRCA2, TP53, PTEN Gold mutations, and/or family history suggestive of the tumor's genetic origin, ${ }^{7,19,20}$ especially for young patients with triple negative tumors and with good response to neoadjuvant therapy. ${ }^{6}$

In a Mayo Clinic study, 214 women classified as high risk and 425 classified as moderate risk underwent bilateral mastectomy. During a 14-year follow-up period, seven breast cancers were diagnosed, which represented a $90 \%$ risk reduction compared to the expected number of neoplasms in this topography. ${ }^{21}$

A prospective analysis in the Netherlands evaluated 583 women with a BRCA mutation between 1980 and 2011, selected from a multicenter cohort. Of these, 242 (42\%) underwent contralateral mastectomy and 341 (58\%) were under observation. $\mathrm{BBC}$ was detected in four patients $(2 \%)$ after contralateral mastectomy and in 64 patients, in the observation group (19\%).2.

The largest prospective analysis of breast cancer after bilateral mastectomy, called the PROSE study and conducted in 2004, evaluated 2,484 women with BRCA1 and BRCA2 mutations and of 22 centers in the United States and Europe. No breast cancer was diagnosed in the 247 women who underwent bilateral mastectomy, whereas 98 breast cancers $(7 \%)$ were diagnosed in the group of those under observation, during the three-year follow-up. ${ }^{23}$

Further studies are needed to better clarify the clinical, familial, histological, and immunohistochemical pattern of bilateral breast carcinomas, which, although rare, are of great clinical importance.

\section{CONCLUSION}

$\mathrm{BBC}$ is rare and is associated with a relevant family history. The most frequent pattern was ductal carcinoma with luminal subtype B. In this sample, the synchronic type was the most common.

\section{AUTHORS' CONTRIBUTIONS}

C.V.P.: conceptualization, research, methodology; data acquisition; statistical analysis, data interpretation, article writing, article review; B.R.B.: data acquisition, data interpretation, article writing; L.R.B.: conceptualization, research and methodology, data interpretation, article review; M.N.A.: data acquisition, data interpretation; V.M.B.: conceptualization, investigation, methodology, data interpretation, article review. 


\section{REFERENCES}

1. Manea E, Munteanu A. Evolution of synchronous bilateral breast carcinoma in a young patient. Rev Med Chir Soc Med Nat Iasi. 2016;120(1):192-6. PMID: 27125095

2. Vuoto HD, García AM, Candás GB, Zimmermann AG, Uriburu JL, Isetta JA, et al. Bilateral breast carcinoma: clinical characteristics and its impact on survival. Breast J. 2010;16(6):625-32. https://doi.org/10.1111/j.15244741.2010.00976.x

3. Gollamudi SV, Gelman RS, Peiro G, Schneider LJ, Schnitt SJ, Recht A, et al. Breast-conserving therapy for stage I-II synchronous bilateral breast carcinoma. Cancer. 1997;79(7):1362-9. https://doi.org/10.1002/(SICI)10970142(19970401)79:7<1362::AID-CNCR14>3.0.CO;2-Y

4. Imyanitov EN, Hanson KP. Molecular pathogenesis of bilateral breast cancer. Cancer Lett. 2003;191(1):1-7. https://doi. org/10.1016/s0304-3835(02)00523-2

5. Kilgore AR, Bell HG, Ahlquist Junior RE. Cancer in the second breast. Am J Surg. 1956;92(2):156-61. https://doi.org/10.1016/ s0002-9610(56)80055-x

6. Mau C, Untch M. Prophylactic surgery: for whom, when and how? Breast Care (Basel). 2017;12(6):379-84. https://doi. org/10.1159/000485830

7. Hunt KK, Euhus DM, Boughey JC, Chagpar AB, Feldman SM, Hansen NM, et al. Society of surgical oncology breast disease working group statement on prophylactic (risk-reducing) mastectomy. Ann Surg Oncol. 2017;24(2):375-97. https://doi. org/10.1245/s10434-016-5688-z

8. Beckmann KR, Buckingham J, Craft P, Dahlstrom JE, Zhang Y, Roder D, et al. Clinical characteristics and outcomes of bilateral breast cancer in an Australian cohort. Breast. 2011;20(2):158-64. https://doi.org/10.1016/j.breast.2010.10.004

9. Chen Y, Thompson W, Semenciw R, Mao Y. Epidemiology of contralateral breast cancer. Cancer Epidemiol Biomarkers Prev. 1999;8(10):855-61. PMID: 10548312

10. Daly MB, Pilarski R, Berry M, Buys SS, Farmer M, Friedman $\mathrm{S}$, et al. NCCN guidelines insights: genetic/familial highrisk assessment: breast and ovarian, version 2.2017. J Natl Compr Canc Netw. 2017;15(1):9-20. https://doi.org/10.6004/ jnccn.2017.0003

11. Hemminki K, Ji J, Försti A. Risks for familial and contralateral breast cancer interact multiplicatively and cause a high risk. Cancer Res. 2007;67(3):868-70. https://doi.org/10.1158/00085472.CAN-06-3854

12. Reiner AS, John EM, Brooks JD, Lynch CF, Bernstein L, Mellemkjær L, et al. Risk of asynchronous contralateral breast cancer in noncarriers of BRCA1 and BRCA2 mutations with a family history of breast cancer: a report from the Women's Environmental Cancer and Radiation Epidemiology Study. J Clin Oncol. 2013;31(4):433-9. https://doi.org/10.1200/ JCO.2012.43.2013
13. Malone KE, Begg CB, Haile RW, Borg A, Concannon P, Tellhed L, et al. Population-based study of the risk of second primary contralateral breast cancer associated with carrying a mutation in BRCA1 or BRCA2. J Clin Oncol. 2010;28(14):240410. https://doi.org/10.1200/JCO.2009.24.2495

14. Metcalfe K, Gershman S, Lynch HT, Ghadirian P, Tung N, KimSing C, et al. Predictors of contralateral breast cancer in BRCA1 and BRCA2 mutation carriers. Br J Cancer. 2011;104(9):138492. https://doi.org/10.1038/bjc.2011.120

15. Adami HO, Bergström R, Hansen J. Age at first primary as a determinant of the incidence of bilateral breast cancer. Cumulative and relative risks in a populationbased case-control study. Cancer. 1985;55(3):643-7. https:// doi.org/10.1002/1097-0142(19850201)55:3<643::aidcncr2820550328>3.0.co;2-1

16. Jobsen JJ, van der Palen J, Ong F, Riemersma S, Struikmans $\mathrm{H}$. Bilateral breast cancer, synchronous and metachronous; differences and outcome. Breast Cancer Res Treat. 2015;153(2):277-83. https://doi.org/10.1007/s10549-015-3538-5

17. Kurian AW, McClure LA, John EM, Horn-Ross PL, Ford JM, Clarke CA. Second primary breast cancer occurrence according to hormone receptor status. J Natl Cancer Inst. 2009;101(15):1058-65. https://doi.org/10.1093/jnci/djp181

18. Kappikeri VK, Kriplani AM. Bilateral synchronous carcinoma breast- a rare case presentation. Springerplus. 2015;4:193. https://doi.org/10.1186/s40064-015-0953-3

19. Mai PL, Best AF, Peters JA, DeCastro RM, Khincha PP, Loud JT, et al. Risks of first and subsequent cancers among TP53 mutation carriers in the National Cancer Institute LiFraumeni syndrome cohort. Cancer. 2016;122(23):3673-81. https://doi.org/10.1002/cncr.30248

20. Carbine NE, Lostumbo L, Wallace J, Ko H. Risk-reducing mastectomy for the prevention of primary breast cancer (Review). Cochrane Database Syst Rev. 2018;4(4):CD002748. https://doi.org/10.1002/14651858.CD002748.pub4

21. Hartmann LC, Schaid DJ, Woods JE, Crotty TP, Myers JL, Arnold PG, et al. Efficacy of bilateral prophylactic mastectomy in women with a family history of breast cancer. N Engl J Med. 1999;340(2):77-84. https://doi.org/10.1056/ NEJM199901143400201

22. Heemskerk-Gerritsen BA, Rookus MA, Aalfs CM, Ausems MG, Collée JM, Jansen L, et al. Improved overall survival after contralateral risk-reducing mastectomy in BRCA1/2 mutation carriers with a history of unilateral breast cancer: a prospective analysis. Int J Cancer. 2015;136(3):668-77. https:// doi.org/10.1002/ijc.29032

23 Rebbeck TR, Friebel T, Lynch HT, Neuhausen SL, van 't Veer L, Garber JE, et al. Bilateral prophylactic mastectomy reduces breast cancer risk in BRCA1 and BRCA2 mutation carriers: the PROSE Study Group. J Clin Oncol. 2004;22(6):1055-62. https:// doi.org/10.1200/JCO.2004.04.188 